\title{
Capacidade funcional e fragilidade de idosos com sequela de tuberculose pulmonar
}

\section{Functional capacity and fragility of the elderly with pulmonar tuberculosis sequelae}

\author{
Bruna D' Paula Souza da Costa' ${ }^{1}$ \\ Luzielma Macêdo Glória ${ }^{2}$ (1) \\ Cleonardo Augusto da Silva ${ }^{3}$ (])
}

\author{
Denise da Silva Pinto 4 \\ Edilene do Socorro Nascimento Falcão Sarges ${ }^{5}$ (1) \\ Rodolfo Gomes do Nascimento ${ }^{6}$ (1)
}

1,3-6Universidade Federal do Pará (Belém). Pará, Brasil. brunadpaulasc@gmail.com, cleonardoaugusto@uol.com.br,denisefisio23@yahoo.com.br, edilene_falcao@hotmail.com, rodgn@hotmail.com ${ }^{2}$ Autora para correspondência. Universidade Federal do Pará (Belém). Pará, Brasil. luzielma96@hotmail.com
RESUMO | INTRODUÇÃO: A tuberculose (TB) é uma doença de caráter infectocontagiosa, de evolução crônica, causada pela Mycobacterium tuberculosis, Uma vez considerada como doença crônica entre idosos por poder gerar sequelas e resultar em comprometimento da capacidade pulmonar, e desta forma contribuir para um quadro clínico de pior prognóstico é essencial estudar a relação da TB com a síndrome da fragilidade e a capacidade funcional em idosos. OBJETIVO: Analisar a capacidade funcional de idosos com seqüela de TB Pulmonar e investigar a ocorrência de fragilidade nesta população. MATERIAIS E MÉTODOS: Trata-se de um estudo transversal com método quantitativo-correlacional, realizado no período de agosto a novembro de 2017, no ambulatório de fisioterapia do Hospital Universitário João de Barros Barreto, Belém/PA Avaliou-se a fragilidade pelo instrumento Tilburg Frailty Indicator (TFI) e a capacidade funcional pelo "Teste de AVD-Glittre" (TGlittre). Utilizou-se a "correlação de Spearman" entre TFI e TGlittre. Adotou-se p-valor $\leq 0,05$. RESULTADOS: Avaliaram-se 24 idosos com diagnóstico clínico de TB Pulmonar, apresentando alterações e/ou sequelas pulmonares. A média de idade foi de $68,37 \pm 6,01$ anos, $66,7 \%$ era do sexo feminino. A presença de fragilidade foi de 70,5\% (IC95\% 48,9-87,4), com predomínio feminino $(82,4 \%)$. A média do tempo no TGlittre foi de $5,77 \pm 1,71$ minutos. Houve correlação moderada positiva entre TFI e TGlittre $(p=0.42, p=0.03)$, CONCLUSÃO: Este estudo identificou a fragilidade e comprometimento funcional em idosos com sequela de TB pulmonar, com prevalência alta de fragilidade se comparada aos outros estudos brasileiros com a população idosa, além de apresenta correlação moderada positiva entre a capacidade funcional e fragilidade, mostrando que essa população merece atenção especial nos programas de reabilitação para a manutenção ou recuperação funcional.

PALAVRAS-CHAVE: Tuberculose pulmonar. Saúde do idoso. Idoso fragilizado. Atividades cotidianas.
ABSTRACT | INTRODUCTION: Tuberculosis (TB) is an infectious and contagious disease, with a chronic evolution that is caused by Mycobacterium tuberculosis, as it is considered a chronic disease among the aged because it can cause sequelae and result in impaired lung capacity and thus contribute to a clinical picture of worse prognosis it is interesting to study the relationship between TB and frailty syndrome and functional capacity in the old age. OBJECTIVE: To analyze the functional capacity of elderly people with pulmonary TB sequelae treated at a pulmonology service in Belém-PA, and to investigate the occurrence of frailty in this population. MATERIALS AND METHODS: This is a crosssectional study with the quantitative-correlational method, conducted from August to November 2017 at the physiotherapy outpatient clinic of the João de Barros Barreto University Hospital, Belém/PA. Frailty by the Tilburg Frailty Indicator (TFI) instrument and functional capacity by the "ADL-Glittre Test" (TGlittre) were evaluated. The "Spearman correlation" between TFI and TGlittre was used. P-value was adopted $\leq 0.05$. RESULTS: The average age was $68,37 \pm 6.01$ years, $66.7 \%$ were female, all individ-uals were literate, the majority with incomplete primary education (45.8\%). The presence of frailty was $70.5 \%(95 \%$ $\mathrm{Cl}$ 48.9-87.4), with a female predominance (82.4\%). The average time on TGlittre was $5,77 \pm 1,71$ minutes. There was a moderate positive correlation between TFI and TGlittre $(p=0.42, p=0.03)$. CONCLUSION: This study identified frailty and functional impairment in the elderly with pulmonary TB sequelae, with a high prevalence of frailty compared to other Brazilian studies with the elderly population, besides presenting a moderate positive correlation between functional capacity and frailty, suggesting that these aspects de-serve special attention in rehabilitation programs for functional maintenance or recovery in this profile of patients.

KEYWORDS: Pulmonary tuberculosis. Elderly health. Frail elderly. Daily activities.

Como citar este artigo: Costa BDPS, Glória LM, Silva CA, Pinto DS Sarges ESNF, Nascimento RG. Capacidade funcional e fragilidade de idosos com sequela de tuberculose pulmonar. Rev Pesqui Fisioter. 2021;11(1):145-154. http://dx.doi.org/10.17267/2238-2704rpf. v11i1.3404 


\section{Introdução}

A tuberculose (TB) é uma doença de caráter infectocontagiosa de evolução crônica, causada pela Mycobacterium tuberculosis e transmitida predominantemente por via aérea, que compromete principalmente os pulmões. Esta enfermidade acomete o mundo há milhares de anos e ainda hoje é considerada um grave problema de saúde pública e uma importante causa de morbimortalidade ${ }^{1,2}$, como demonstram os dados da OMS, que revelam que aproximadamente 10,4 milhões de pessoas adoeceram com TB em 2016 mundialmente, com notificação de 6,3 milhões de casos novos e cerca de 1,5 milhões de óbitos, e estima-se que quase a sua totalidade ocorra nos países em desenvolvimento ${ }^{3}$, estando o Brasil entre os 22 países com maior prevalência ${ }^{4}$, e, entre os estados da região Norte, o Pará ocupou o $1^{\circ}$ lugar em notificações de casos novos no período compreendido entre 1990 a 2014 .

No Brasil, a transição demográfica e epidemiológica ocorre de forma atípica, caracterizando-se tanto pela reemergência ou presença constante das doenças infecciosas e parasitárias quanto pela maior prevalência de doenças e agravos crônicos não transmis-

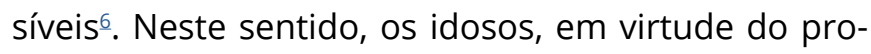
cesso de senescência dos sistemas biológicos, como o imunológico e respiratório, encontram-se frequentemente mais vulneráveis à ação do $M$. tuberculosis, tanto à infecção exógena quanto para a reativação de focos contendo bacilos em estado de latência ${ }^{7}$. Além disso, essa população tem grande probabilidade de apresentar sequelas da TB pulmonar caso tenha sofrido exposição durante a infância, período no qual a prevalência da doença era alta e os esquemas terapêuticos menos eficazes ${ }^{8}$.

A TB pode levar a uma série de consequências prejudiciais, dentre estas, a presença de lesões pulmonares extensas pode ser um fator preditor de invalidez permanente devido à insuficiência respiratória secundária a destruição tecidual, Cor Pulmonale, além de predisposição a infecções oportunistas, o que resulta em comprometimento na capacidade funcional e na qualidade de vida, além de se tornar um dos fatores de risco implicados na mortalidade pela doença?

Estudos de Chaves et al. $\stackrel{?}{\text { e L Lourenço e Lopes }}{ }^{\stackrel{8}{ }}$ demonstram que indivíduos idosos que morreram ou foram internados com ou por tuberculose possuíram maior chance de não notificação que adultos mais jovens, reforçando a hipótese de barreira de acesso dos mais velhos ao Programa de Controle de Tuberculose e uma possível cronificação da doença, podendo assim ser considerada mais uma doença pulmonar crônica entre os idosos, e uma vez que a doença pulmonar crônica participa significativamente de um quadro clínico pior, é interessante estudar sua relação com a síndrome da fragilidade, que apresenta como manifestações dominantes a diminuição da força muscular, sensação de cansaço, perda involuntária de peso, lentidão e inatividade ${ }^{10,11}$, e se associa aos desfechos adversos, tais como declínio e incapacidade funcional e à restrição de atividades entre os idosos ${ }^{12}$.

A capacidade funcional, por sua vez, é definida como a ausência de dificuldades no desempenho de gestos específicos e de certas atividades da vida cotidiana, que engloba todas as funções do corpo e a capacidade do indivíduo em realizar tarefas relevantes da sua rotina diária ${ }^{11}$. Por estes aspectos serem imprescindíveis, instrumentos capazes de simular as situações cotidianas destes pacientes são de grande importância e devem ser incluídos na sua avaliação. No entanto, desconhecem-se estudos que tenham realizados testes ou instrumentos de avaliação funcional específicos para pacientes com sequela de TB pulmonar, havendo uma escassez de informações na literatura sobre a capacidade funcional desses indivíduos, também são escassos estudos que avaliem a fragilidade em idosos com sequela de TB, assim como bem poucos foram encontrados com a aplicação da TFI a população de idosos brasileiros.

Diante do exposto, esta pesquisa se propôs a analisar a capacidade funcional de idosos com sequela de Tuberculose Pulmonar atendidos em um serviço de pneumologia de Belém-PA, bem como investigar a ocorrência de fragilidade nesta população.

\section{Material e métodos}

Trata-se de um estudo transversal com método quantitativo-correlacional, realizado de acordo com a Resolução 466/2012 para pesquisas envolvendo seres humanos, aprovado pelo Comitê de Ética em Pesquisa do Hospital Universitário João de Barros Barreto da Universidade Federal do Pará (CEP/HUJBB/UFPA), sob o parecer $n^{\circ}$ CA 64545617.7.0000.0017, que foi realizado no período de agosto a novembro de 2017. 
A amostragem deu-se por conveniência, sendo a amostra constituída por indivíduos de ambos os sexos, com idade igual ou superior a 60 anos, com diagnóstico clínico de TB Pulmonar, apresentando alterações e/ou sequelas pulmonares. Os idosos eram encaminhados para a participação na pesquisa pela equipe médica dos ambulatórios de pneumologia do HUJBB. Cabe salientar que os idosos podiam ter ou não outras doenças pulmonares.

Foram excluídos do estudo os idosos que apresentaram dispneia severa na escala de Borg modificada (5 a 10); queixas álgicas intensas $(8,9,10)$ na Escala visual Analógicas; indivíduos cadeirantes ou com algum outro comprometimento musculoesquelético e/ ou neuromusculares que limitasse a realização do teste funcional, sendo admitido fazer uso de recursos auxiliares de correção visual ou auditiva; e ainda os que apresentaram déficit de compreensão.

Após a assinatura do Termo de Consentimento Livre e Esclarecido, os idosos responderam a um formulário produzido pelos pesquisadores contendo dados como: idade, sexo, escolaridade, estado civil, interrupção do tratamento, realização do tratamento farmacológico logo que descobriu, comorbidades, idade em que adquiriu TB, duração do tratamento, peso, altura, IMC e sensação de dispneia, seguida da avaliação de fragilidade por meio da versão brasileira do instrumento Tilburg FrailtyIndicator (TFI), validado por Santiago e Mattos $\frac{14}{}$, que é constituída por 15 questões objetivas, auto avaliativas, distribuídas nos domínios "físico" (questões referentes à auto avaliação de saúde, perda de peso, capacidade de caminhada e equilíbrio, déficit auditivo e visual, fraqueza nas mãos e percepção de cansaço), "psicológico" (relacionado à condição de memória, sensação de tristeza, nervosismo ou ansiedade e enfrentamento de problemas) e "social" (questões referente a morar sozinho, se sente falta de pessoas ao seu lado ou de apoio), às quais foi atribuída a pontuação "zero" ou "um".

Os escores desses domínios variam, respectivamente, de 0-8, 0-4 e 0-3. O escore final pode variar entre zero e 15 pontos, e quanto maior a pontuação, maior o grau de fragilidade ou, alternativamente, escores $\geq 5$ pontos indicam o indivíduo como frágil. Nesta pesquisa, considerou-se frágil o indivíduo que obteve escore total igual ou superior a cinco no TFI ${ }^{14}$.
Posteriormente, a condição funcional foi avaliada por meio do "Teste de AVD-Glittre" (TGlittre), que foi desenvolvido e validado para pacientes com DPOC $\frac{15}{5}$, mas que recentemente tem sido aplicado a variados perfis de pacientes. Ele envolve um conjunto de tarefas em um circuito que consiste no ato de levantar-se de uma cadeira, caminhar por uma pista de 10 metros carregando uma mochila nas costas com peso de 2,5 Kg para mulheres e $5 \mathrm{Kg}$ para homens (o que se assemelha ao peso de um equipamento de oxigênio suplementar), subir e descer uma escada com dois degraus (com altura de $17 \mathrm{~cm}$ e largura de 27 $\mathrm{cm}$ cada degrau) interposta na metade da pista, no final do trajeto o indivíduo se depara com uma estante e deve transferir três objetos um a um (com 1 $\mathrm{Kg}$ de peso cada) da prateleira alta (na altura de seus ombros) para prateleira baixa (na altura da cintura pélvica), depois até o chão, retornar novamente com os objetos para a prateleira baixa e finalmente para a prateleira alta. Em seguida, o paciente retorna fazendo o mesmo percurso até sentar-se outra vez na cadeira; imediatamente reinicia outra volta percorrendo o mesmo circuito de AVD até completar cinco voltas. O paciente é incentivado a realizar a tarefa o mais rápido possível podendo parar para descansar, porém com o cronometro acionado ${ }^{15}$.

A pressão arterial foi aferida por meio do esfigmomanômetro Premuium ${ }^{\circledR}$ e estetoscópio Littmann ${ }^{\circledR}$, a saturação periférica de oxigênio e frequência cardíaca foram aferidas pelo oxímetroOxi-Goße o nível de dispneia foi obtido por meio da "Escala de Borg modificada ${ }^{16}$. Tais avaliações foram realizadas no início e no final do desempenho do teste TGlittre. O tempo total para completar o teste foi registrado e utilizado como desfecho. Quanto maior o tempo para desempenhar o teste, pior é a capacidade funcional do paciente ${ }^{15}$.

Mediante os dados obtidos, o processamento foi iniciado com a entrada dos dados no Microsoft Windows Excel $2007 \circledast$. A análise estatística descritiva das variáveis categóricas foi apresentada em número absoluto, frequência relativa e intervalo de confiança de 95\% (IC95\%). Já as numéricas foram apresentadas em média, desvio padrão, máximo e mínimo. Para as correlações entre TGlittre e TFI, aplicou-se o "coeficiente de correlação de Spearman", adotando o nível de significância de $5 \%(p \leq 0,05)$. 
Os programas utilizados para a análise foram o Software Epi Info 7.1.5 e o programa Bioestat 5.3, a partir dessa análise foram gerados os gráficos e tabelas.

\section{Resultados}

Foram encaminhados 31 pacientes com sequela de TB pulmonar no período da realização do estudo, no entanto, 7 foram excluídos desses 3 pela idade, pois não tinham 60 anos completos, 2 por não terminarem avaliação no período da pesquisa, pois moravam no interior, e 2 por desistência da pesquisa.

Desta maneira, a pesquisa obteve uma amostra de 24 idosos, com média de idade de $68,37 \pm 6,01$ anos, sendo $62,5 \%$ do sexo feminino e 50,0\% casados. Quanto ao nível de escolaridade, todos os indivíduos eram alfabetizados, sendo que a maioria frequentou apenas os primeiros anos escolares, possuindo o ensino fundamental incompleto com 45,8\% da amostra (Tabela 1).

A média de idade com a qual apresentaram a doença foi de $35,16 \pm 4,6$ anos, e quanto à realização do tratamento da TB, a maior parte dos indivíduos, 79,2\% da amostra, concluiu os esquemas medicamentosos sem interrupção (Tabela 1), com média da duração do tratamento de 6,75 $\pm 5,44$ meses, sendo que grande parte deles iniciaram logo que diagnosticado, além disso, a maior parte, 66,7\%, não tinha comorbidades associadas (Tabela 1).

Tabela 1. Caracterização dos idosos com sequela de tuberculose pulmonar atendidos no HUJBB ( $n=24)$ de acordo com as variáveis categóricas. Belém, PA, 2017

\begin{tabular}{|c|c|c|c|}
\hline Variáveis & $\begin{array}{c}\text { Número } \\
\text { absoluto }\end{array}$ & $\begin{array}{l}\text { Frequência } \\
\text { relativa (\%) }\end{array}$ & IC95\% \\
\hline \multicolumn{4}{|l|}{ Sexo } \\
\hline Masculino & 9 & 37,5 & $18,8-59,4$ \\
\hline Feminino & 15 & 62,5 & $40,6-81,2$ \\
\hline \multicolumn{4}{|l|}{ Estado civil } \\
\hline Casado (a) & 12 & 50,0 & $29,1-70,9$ \\
\hline Solteiro (a) & 8 & 33,3 & $15,6-55,3$ \\
\hline Viúvo (a) & 4 & 16,7 & $4,7-37,4$ \\
\hline Ensino fundamental incompleto & 11 & 45,8 & $25,6-67,2$ \\
\hline Ensino fundamental completo & 9 & 37,5 & $18,8-59,4$ \\
\hline Ensino médio completo & 3 & 12,5 & $2,7-32,4$ \\
\hline Ensino superior completo & 1 & 4,2 & $0,1-21,1$ \\
\hline \multicolumn{4}{|l|}{ Interrupção do tratamento } \\
\hline $\operatorname{Sim}$ & 4 & 16,7 & $4,7-37,4$ \\
\hline Não & 19 & 79,2 & $57,8-92,9$ \\
\hline Não tratou & 1 & 4,2 & $0,1-21,1$ \\
\hline \multicolumn{4}{|l|}{ Tratou logo quando descobriu } \\
\hline Sim & 20 & 83,3 & $62,6-95,3$ \\
\hline Não & 4 & 16,7 & $4,7-37,4$ \\
\hline \multicolumn{4}{|l|}{ Comorbidades* } \\
\hline Sim & 8 & 33,3 & $15,6-55,3$ \\
\hline Não & 16 & $66,7 \%$ & $44,7-84,4$ \\
\hline
\end{tabular}

Comorbidades* (hipertensão, diabetes, asma, doença pulmonar obstrutiva crônica). 
Os dados antropométricos mostram uma média de altura de 1,53 $\pm 0,09 \mathrm{~m}$, peso de $62,50 \pm 13,75 \mathrm{~kg}$ e um IMC de $26,54 \pm 5,23 \mathrm{~kg} / \mathrm{m} 2$, a sensação de dispneia no dia da avaliação apresentou uma média de 2,12 $\pm 0,67$. A fragilidade analisada pelo TFI obteve um escore total de $6,12 \pm 2,90$ pontos, já a capacidade funcional avaliada pelo TGlittre obteve uma média de 5,75 $\pm 1,71$ em minutos.

A prevalência de fragilidade estimada para a amostra, segundo o ponto de corte proposto para o TFI, foi de $70,5 \%$ (IC95\% 48,9-87,4) e o item mais referido nesta escala foi a sensação de cansaço como aspectos que causam problemas no dia a dia, que equivaleu a $75 \%$ das respostas positivas, sendo que $94,44 \%$ dos caracterizados como frágeis relataram cansaço.

Tabela 2. Caracterização dos idosos com sequela de tuberculose pulmonar atendidos no HUJBB ( $n=24)$ de acordo com as variáveis numéricas. Belém, PA, 2017

\begin{tabular}{cccc}
\hline Variáveis & Média & Desvio Padrão & Máximo-Mínimo \\
\hline Antropometria & 1,53 & & \\
Altura $(\mathrm{m})$ & 62,50 & $\pm 0,09$ & $1,70-1,39$ \\
Peso $(\mathrm{kg})$ & 26,54 & $\pm 13,75$ & $86,90-39,30$ \\
IMC $\left(\mathrm{kg} / \mathrm{m}^{2}\right)$ & & & $38,73-17,99$ \\
Sensação de dispnéia & 2,12 & $\pm 0,67$ & $4-1$ \\
Escala Borg modificada & & & $7-1$ \\
Fragilidade - TFI & 3,75 & $\pm 1,87$ & $4-0$ \\
Domínio físico & 1,50 & $\pm 1,10$ & $2-0$ \\
Domínio psicológico & 0,91 & $\pm 0,65$ & $13-1$ \\
Domínio social & 6,12 & $\pm 2,90$ & $10,20-3,80$ \\
$\quad$ Escore total & & & $\pm 1,71$ \\
Capacidade funcional & 5,75 & & \\
TGlittre (minutos) &
\end{tabular}

A análise de correlação mostrou que o escore total obtido no TFI apresentou correlação positiva moderada com o tempo de execução do TGlittre com valor de " $p$ " estatisticamente significante ( $\rho=0,42$, p-valor=0,03), (Figura 1).

Figura 1. Correlação de Spearman entre tempo para realização do TGlittre e escore total obtido no TFI por pacientes idosos com seqüela de tuberculose pulmonar do HUJBB. Belém, PA, 2017

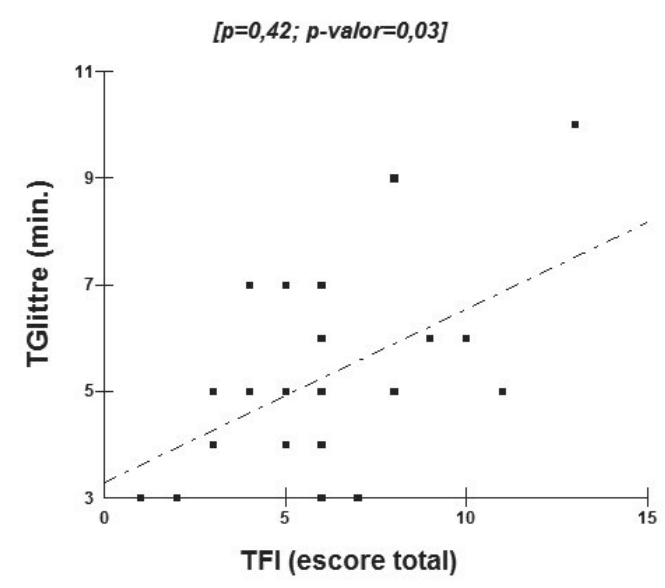


Nas figuras seguintes, foram feitas as correlações com cada domínio do TFI; na figura 2, observa-se uma correlação positiva fraca entre o domínio físico e tempo de execução do TGlittre, sendo que essa correlação não foi estatisticamente significante $(\rho=0,33$, p-valor $=0,10)$.

Figura 2. Correlação de Spearman entre o tempo para realização do TGlittre e o domínio físico da TFI por pacientes idosos com seqüela de tuberculose pulmonar do HUJBB. Belém, PA, 2017.

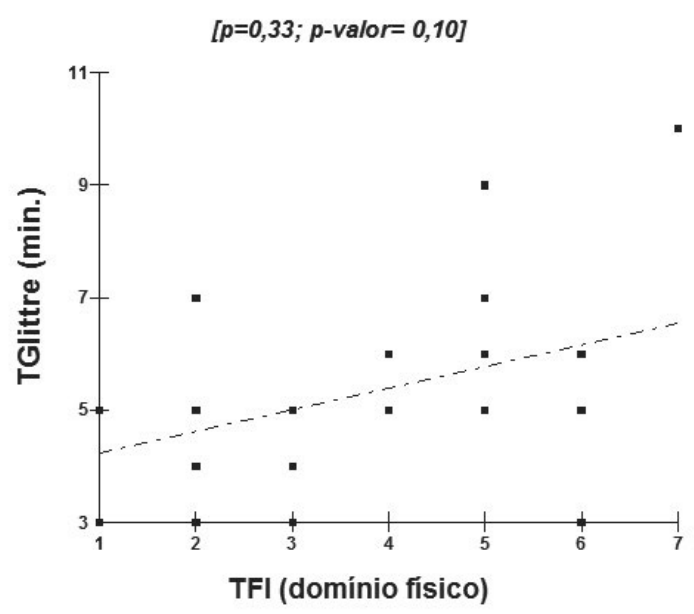

Em relação à figura 3, podemos ver também uma correlação positiva fraca entre o domínio psicológico e tempo de execução do TGlittre, sendo que essa correlação não foi estatisticamente significante $(\rho=0,34$, $p$-valor= 0,09$)$.

Figura 3. Correlação de Spearman entre o tempo para realização do TGlittre e o domínio psicológico da TFI por pacientes idosos com sequela de tuberculose pulmonar do HUJBB. Belém, PA, 2017

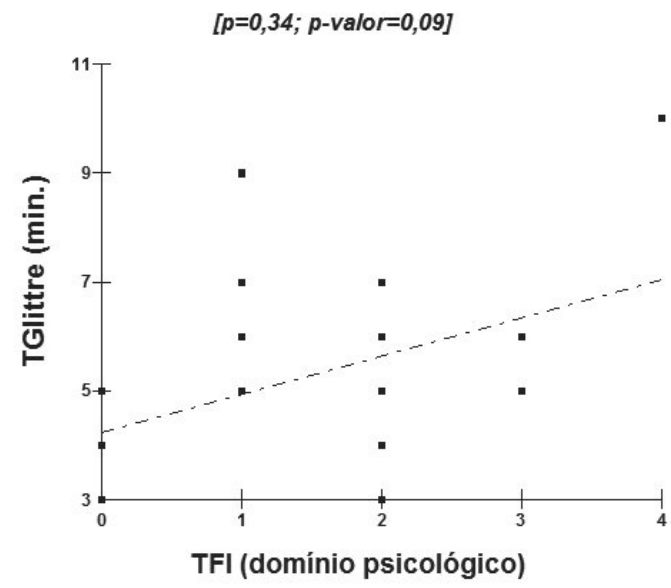

Por fim, na figura 4, pode-se observar que a correlação entre o domínio social e o tempo de execução do TGlittre apresentou correlação positiva moderada com valor de " $p$ " estatisticamente significante $(\rho=0,46, p$-valor= 0,02$)$. 


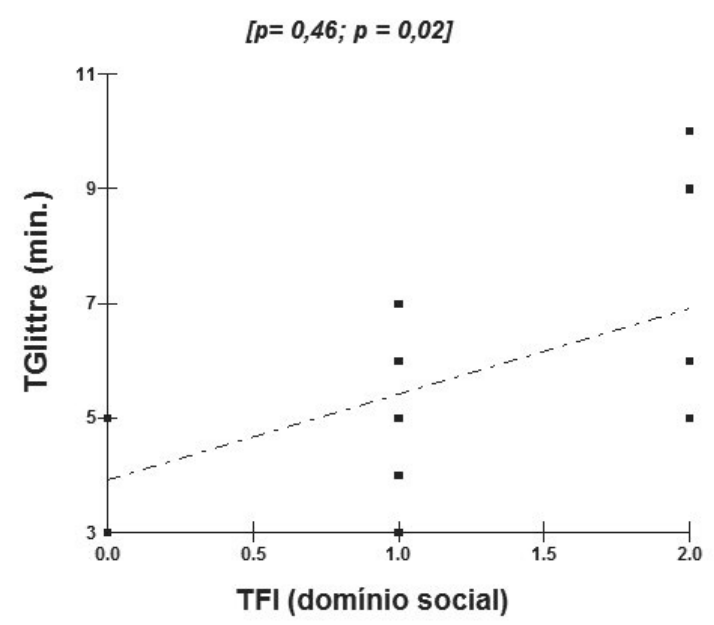

\section{Discussão}

Na presente pesquisa pode-se observar que a capacidade funcional estava prejudicada, uma vez que o tempo médio para executar foi maior com 5,75 $\pm 1,71$ minutos. Além disso, a fragilidade teve uma média alta de $6,12 \pm 2,90$ no presente estudo, e as correlações entre o tempo de execução do TGlittre e o escorre total da TIF mostrou que quanto maior o tempo para executar o teste da capacidade funcional, maior o escore total da fragilidade, tendo assim uma correlação positiva moderada com significância estatística $(\rho=0,42, p$-valor $=0,03)$.

Um dos achados do presente estudo foi à prevalência de fragilidade segundo o ponto de corte proposto para o instrumento de rastreio utilizado, que foi equivalente a $70,5 \%$ da amostra, com média de escore total de 6,12 $\pm 2,9$ pontos, demonstrando-se elevada ao se comparar com outros estudos brasileiros. Em uma pesquisa realizada com uma população de idosos institucionalizados, a prevalência de fragilidade analisada por meio do TFI foi de $52 \%$, com média do escore total de 4,9 $\pm 2,5$ pontos $^{14}$, já em um estudo com idosos comunitários a prevalência foi de $48,5 \%$, com média de 4,77 $\pm 2,7$ pontos ${ }^{17}$. Por outro lado, o estudo de Fried et al. ${ }^{18}$ observou que embora pessoas idosas possam sofrer fragilidade sem doença associada, pode existir uma sobreposição entre a fragilidade e comorbidades, especialmente com doenças crônicas, isso pode ser verificado pela pesquisa atual, que estudou a fragilidade em idosos com sequela de TB.

Sabe-se também que o grau de capacidade funcional em idosos é muitas vezes agravado pelo surgimento de doenças crônicas e seus efeitos nocivos cumulativos durante o processo de envelhecimento, aumentando o risco de eventos adversos à saúde, tendo em vista estes aspectos, o achado de prevalência elevada de fragilidade no presente estudo pode sugerir relação com sequelas de TB pulmonar, uma vez que estas que podem acarretar em um quadro de comprometimento pulmonar crônico e, por sua vez, tais agravos resultarem em maior probabilidade do idoso se tornar frágil, além disso, o estudo indica relação da tuberculose com o quadro de sarcopenia patológica, um dos achados comuns nos quadros de fragilidade ${ }^{10}$.

Para esta população, seja a TB resultante de reativação endógena ou da reinfecção exógena (novo contágio), a síndrome de fragilidade poderia ser tanto uma expressão de formas primárias, onde a redução da reserva funcional associada ao envelhecimento seria o principal mecanismo fisiopatológico, quanto da forma secundária, onde o aparecimento de determinadas morbidades acelerariam as perdas funcionais, alcançando mais rapidamente 0 nível crítico a partir do qual apareceriam os sinais, sintomas e complicações frequentes entre os idosos frágeis ${ }^{19}$. 
Apesar de não haver consenso sobre a definição da síndrome da fragilidade ${ }^{10,11}$, alguns aspectos que a caracterizam foram evidenciados na população estudada, na qual o relato de cansaço foi o mais referido no TFI como um aspecto que causa problemas no dia a dia, outra questão bastante referida foi a dificuldade ao caminhar. Cabe ressaltar que esses aspectos podem ser evidenciados em pacientes pós-tuberculose em decorrência de alterações respiratórias ou sistêmicas, pois estes podem apresentar limitada tolerância ao exercício favorecendo o descondicionamento e comprometimento sistêmico com prejuízo as atividades de vida diária ${ }^{20}$, além disto, é sabido que a força muscular respiratória está relacionada com a redução da mobilidade em idosos, independentemente do nível de atividade física e da força dos membros inferiores ${ }^{21}$.

Quanto à avaliação da capacidade funcional através do TGlittre, o tempo médio obtido por esta população para a execução do teste foi superior ao alcançado por indivíduos com DPOC. A exemplo do estudo de Corrêa et al. $\underline{22}$, que fez correlação entre o TC6min e o TGlittre em idosos saudáveis e idosos com DPOC (idade: $64 \pm 10$; VEF1\%pred.: $38,1 \pm 11,8$ ), em que o grupo DPOC apresentou pior desempenho comparado ao de saudáveis no TGlittre $(5,29 \pm 2,9$ min. vs 3,3 $\pm 0,3$ min., $p<0,05$ ) e também quando comparado aos estudos de Gulart et al. ${ }^{15}$, em que os indivíduos com DPOC (idade $=63,9 \pm 8,1$ ) alcançaram tempo médio igual a $4,5( \pm 1,2)$ minutos para realizar o teste.

Embora a maioria dos estudos $15,23,24$ utilizando o TGlittre tenha sido realizada com pacientes com quadro de DPOC, uma vez que o instrumento foi elaborado para este perfil populacional, torna-se pertinente a sua utilização para avaliação da população alvo deste estudo, bem como a comparação entre os resultados encontrados nesta populações, pois apesar das diferenças entre suas fisiopatologias primárias, observam-se semelhanças entre os dois grupos, tais como dispneia e a limitação da capacidade funcional, decorrente de alterações estruturais, de parênquima pulmonar, padrão ventilatório, entre outras 5 15,23,24.
No estudo, Gulart et al. $\frac{15}{}$ também constataram que o TGlittre, quando comparado ao TC6, apresentou-se como melhor preditor da percepção de limitação funcional na população estudada. Ressalta-se que a capacidade de execução das atividades de vida diária (AVD) são melhores preditas por meio de testes globais do que por testes focados em componentes isolados da atividade funcional, sendo este instrumento uma boa alternativa de avaliação funcional por ser constituído de atividades que simulam as do cotidiano e envolvem tanto membros inferiores quanto superiores ${ }^{23}$.

A correlação entre os resultados obtidos no TFI e TGlittre indicaram que quanto maior a pontuação total no TFI (maior grau de fragilidade) maior o tempo dispendido para a execução do teste funcional. Este resultado corrobora com os estudos de Fried et al18 que afirmam que a incapacidade funcional, fragilidade e comorbidades são entidades clínicas distintas que não ocorrem necessariamente associadas, mas interagem entre si e, segundo este autor, a fragilidade e a comorbidade predizem a incapacidade funcional e esta, por sua vez, pode agravar a fragilidade e a comorbidade, enquanto esta última pode contribuir ao menos aditivamente no desenvolvimento da fragilidade ${ }^{11,18}$.

Além disso, foram feitas as correlações entre tempo de execução do TGlittre com cada domínio do TFI, constatou-se uma correlação positiva fraca entre o domínio físico e psicológico e uma correlação moderada no domínio social, sendo essas correlações pioneiras encontradas no presente estudo.

Algumas limitações deste estudo devem ser esclarecidas, uma delas é número reduzido da amostra, pois poderia ter interferido no poder estatístico e ser responsável pela ausência de algumas associações. Entretanto, importantes associações foram demonstradas, respondendo positivamente às questões primárias do presente estudo. Além disso, por se tratar de uma amostragem não probabilística por conveniência, o estudo não permite a generalização dos resultados para todos os idosos. Por fim, o delineamento do estudo foi transversal, o que não permitiu estabelecer uma relação de causa e efeito entre as variáveis investigadas e o desfecho do estudo. 
Este estudo contribuiu para identificação de fragilidade e comprometimento funcional em idosos com sequela de TB pulmonar e, ao que parece, foi pioneiro em realizar a avaliação deste perfil populacional por meio dos instrumentos TGlittre e TFI, bem como em correlacioná-los. Desta forma, os resultados desta pesquisa cooperam para a prática clínica, dando maior suporte à aplicação destes instrumentos para a avaliação de populações similares. Ressalta-se que são necessários mais estudos que se dediquem a avaliar aspectos funcionais e de fragilidade em um número maior de indivíduos, bem como a responsividade dessas avaliações a intervenções, como a reabilitação pulmonar.

\section{Conclusão}

Este estudo analisou que a capacidade funcional de idosos com sequela de TB Pulmonar se encontrava prejudicada, conforme notado pelo tempo para execução do TGlittre, que foi superior ao obtido tanto por indivíduos saudáveis quanto com DPOC, indicando maior comprometimento.

Bem como a ocorrência de fragilidade nesta população, que foi relativamente alta se comparada a outros estudos brasileiros, sugerindo que o quadro de sequela de TB pulmonar pode estar relacionado, no entanto, como esta síndrome apresenta caráter multifatorial, é necessário que mais estudos sejam feitos levando em consideração maior abrangência de aspectos.

Identificou-se também uma correlação moderada positiva entre a capacidade funcional e escore total de fragilidade, apontando que estes pacientes merecem atenção especial nos programas de reabilitação tanto para a manutenção, como para a recuperação funcional.

\section{Agradecimentos}

Agradecemos também ao André, do Centro de estudos do HUJBB, por nos ceder o espaço para realizar o TGlittre e por sempre estar disponível.

\section{Contribuições dos autores}

Costa BDOS participou da concepção, delineamento, busca e análise estatística dos dados da pesquisa, interpretação dos resultados, redação do artigo científico. Glória LM participou da concepção, delineamento, coleta de dados, redação do artigo científico. Silva CA participou da coleta de dados, ajudou na classificação dos pacientes que tinham a sequela da tuberculose, ajudou a escrever o método da pesquisa. Pinto DS, ajudou a escrever o método da pesquisa, participou da análise estatística dos resultados da pesquisa. Sarges ESNF participou da redação dos resultados e a discussão. Nascimento RG orientou a pesquisa e revisou criticamente o manuscrito.

\section{Conflitos de interesses}

Nenhum conflito financeiro, legal ou político envolvendo terceiros (governo, empresas e fundações privadas, etc.) foi declarado para nenhum aspecto do trabalho submetido (incluindo, mas não se limitando a subvenções e financiamentos, participação em conselho consultivo, desenho de estudo, preparação de manuscrito, análise estatística, etc.).

\section{Referências}

1. Couto DS, Carvalho RN, Azevedo EB, Moraes MN, Pinheiro $P G O D$, Faustino EB. Fatores determinantes para o abandono do tratamento da tuberculose: representações dos usuários de um hospital público. Saúde debate. 2014;38(102):572-81. http:// dx.doi.org/10.5935/0103-1104.20140053

2. Chaves EC, Carneiro ICRS, Santos MIOI, Sarges NA, Neves EOS. Aspectos epidemiológicos, clínicos e evolutivos da tuberculose em idosos de um hospital universitário em Belém, Pará. Rev. bras. geriatr. Gerontol. 2017;20(1):45-55. http://dx.doi. org/10.1590/1981-22562017020.160069

3. World Health Organization. Global TB report 2017 [Internet]. Geneva: World Health Organization; 2017. Disponível em: https:// www.who.int/tb/publications/global_report/gtbr2017_main_text. pdf?u\%20a=1

4. Ministério da Saúde (Brasil). Brasil Livre da Tuberculose: Plano nacional pelo fim da tuberculose como problema de saúde pública [Internet]. Brasília: Ministério da saúde; 2017. Disponível em: http://portalarquivos2.saude.gov.br/images/pdf/2017/ junho/29/plano_nacional_tb_web.pdf

5. Ministério da Saúde (Brasil). Série histórica de número de casos novos de tuberculose, Brasil, Regiões e Unidades Federadas de residência por ano diagnóstico (1990 a 2014) [Internet]. Brasília: Ministério da Saúde; 2016. Disponível em: http://portalarquivos. saude.gov.br/images/PDF/2015/abril/10/Serie-historica-donumero-de-casos-novos-de-tuberculose-Brasil-Regioes-eUnidades-Federadas-de-residencia-por-ano-diagn--stico1990-a-2014.pdf 
6. Pereira RA, Alves-Souza RA, Vale JS. O processo de transição epidemiológica: uma revisão de literatura. Rev Cient Fac Educ e Meio Ambient [Internet]. 2015;6(1):99-108. Disponível em: http:// www.faema.edu.br/revistas/index.php/Revista-FAEMA/article/ view/322

7. Ghweil AA, Alsenbsy MAR, Badawy M, Abdelkarem MM, Helal MM. Pattern of Tuberculosis in Elderly Patients in Egypt. J Trop Dis. 2018;6(2):262. http://dx.doi.org/10.4172/2329-891X.1000262

8. Lourenço RA, Lopes AJ. Tuberculose no Idoso. Rev. HUPE [internet]. 2006;5(2):90-5. Disponível em: https://www.epublicacoes.uerj.br/index.php/revistahupe/article/view/9214/7097

9. Cruz RCS, Albuquerque MFPM, Campelo ARL, Silva EJC, Mazza E, Menezes RC, et al. Tuberculose Pulmonar: Associação entre extensão de lesão pulmonar residual e alteração da função pulmonar. Rev Assoc Med Bras. 2008;5(54):406-10. https://doi. org/10.1590/S0104-42302008000500012

10. Cabrera AJR. Fragilidad y enfermedades crónicas en los adultos mayores. Med Int Mex [Internet]. 2011;27(5):455-62. Disponível em: https://www.medigraphic.com/pdfs/medintmex/ mim-2011/mim115g.pdf

11. Certo A, Sanchez K, Galvão A, Fernandes H. A síndrome da fragilidade nos idosos: revisão da literatura. Actas gerentol [Internet]. 2016;2(1):1-11. Disponível em: https://bibliotecadigital. ipb.pt/bitstream/10198/12983/1/fragilidade\%20\%20no\%20idoso. pdf

12. Stein CS. Relação entre doença pulmonar crônica referida, fragilidade e fatores associados em idosos comunitários: dados do FIBRA - Unicamp [dissertação] [Internet]. Campinas: Unicamp; 2012. Disponível em: http://repositorio.unicamp.br/bitstream/ REPOSIP/310910/1/Stein_CristianeSerafim_M.pdf

13. Santos CLS, Santos FC, Gomes Neto M, Silva GMP, Morais LH, Nazaré MA. Avaliação da capacidade funcional em pacientes com tuberculose pulmonar. Fisioter Bras [Internet]. 2012;13(1):4-8. Disponível em: https://portalatlanticaeditora.com.br/index.php/ fisioterapiabrasil/article/download/442/908

14. Santiago LM, Mattos IE. Prevalência e fatores associados à fragilidade em idosos institucionalizados das regiões Sudeste e Centro-Oeste do Brasil. Rev. Bras. Geriatr. Gerontol. 2014;17(2):327-37. https://doi.org/10.1590/S180998232014000200010

15. Gulart AA, Santos K, Munari AB, Karloh M, Cani KC, Mayer AF. Relação entre a capacidade funcional e a percepção de limitação em atividades de vida diária de pacientes com DPOC. Fisioter Pesq [Internet]. 2015;22(2):104-11. Disponível em: https://www. scielo.br/scielo.php?pid=S1809-29502015000200104\&script=sci abstract\&tlng $=p t$
16. American Thoracic Society Statement. ATS statement: Guidelines for the six-minute walk test. Am J Respire Crit Care Med. 2002;166(1):111-7. https://doi.org/10.1164/ ajrccm.166.1.at1102

17. Ferreira PSA, Teófilo RR, Tomaz AF. Estudo do déficit cognitivo e sua correlação com a fragilidade em idosos da cidade de Campina Grande. Revista saúde \& ciência [Internet]. 2015;4(3):6471. Disponível em: https://docplayer.com.br/17704235-Revistasaude-ciencia-online.html

18. Fried L, Tangen CM, Walston J, Newman AB, Hirsch,C, Gottdiener J, et al. Frailty in older adults: evidence for a phenotype. J Gerontol A Biol Sci Med Sci. 2001;56(3):146-56. https://doi.org/10.1093/gerona/56.3.m146

19. Lourenço RA. A síndrome de fragilidade no idoso: marcadores biológicos. Rev HUPE [Internet]. 2008;7(1):21-9. Disponível em: https://www.researchgate.net/publication/265468188 A sindrome_de_fragilidade_no_idoso

20. Naso FC, Pereira JS, Schuh SJ, Unis G. Avaliação funcional em pacientes com sequela pulmonar de tuberculose. Rev Port Pneumol. 2011;17(5):216-21. https://doi.org/10.1016/j. rppneu.2011.06.010

21. Parentoni A, Lustosa L, Santos K, Sá L, Ferreira F, Mendonça V. Comparação da força muscular respiratória entre os subgrupos de fragilidade em idosas da comunidade. Fisioter Pesqui. 2013;20(4):361-6. https://doi.org/10.1590/S1809$\underline{29502013000400010}$

22. Corrêa KS, Karloh M, Martins LQ, Santos K, Mayer AF. O teste de AVD-Glittre é capaz de diferenciar a capacidade funcional de indivíduos com DPOC da de saudáveis. Rev Bras Fisioter. 2011;15(6):467-73. https://doi.org/10.1590/S1413$\underline{35552011005000034}$

23. Valadares YD, Corrêa KS, Silva BO, Araujo CLP, Karloh M, Mayer AF. Aplicabilidade de Testes de Atividades de Vida Diária em Indivíduos com Insuficiência Cardíaca. Rev Bras Med Esporte. 2011;17(5):310-4. https://doi.org/10.1590/S1517$\underline{86922011000500003}$

24. World Health Organization. Global tuberculosis control: surveillance, planning, financing [Internet]. Geneva: World Health Organization; 2008. Disponível em: https://apps.who.int/iris/ handle/10665/43831 